\title{
Does Dormancy increase Fitness of Bacterial Populations in Time-Varying Environments?
}

\author{
Tufail Malik* Hal L. Smith*
}

November 26, 2007

\begin{abstract}
A simple family of models of a bacterial population in a time varying environment in which cells can transit between dormant and active states is constructed. It consists of a linear system of ordinary differential equations for active and dormant cells with time-dependent coefficients reflecting an environment which may be periodic or random, with alternate periods of low and high resource levels. The focus is on computing/estimating the dominant Lyapunov exponent, the fitness, and determining its dependence on various parameters and the two strategies - responsive and stochastic - by which organisms switch between dormant and active states. A responsive switcher responds to good and bad times by making timely and appropriate transitions while a stochastic switcher switches continuously without regard to the environmental state. The fitness of a responsive switcher is examined and compared with fitness of a stochastic switcher, and with the fitness of a dormancy-incapable organism. Analytical methods show that both switching strategists have higher fitness than a dormancyincapable organism when good times are rare and that responsive switcher has higher fitness than stochastic switcher when good times are either rare or common. Numerical calculations show that stochastic switcher can be most fit when good times are neither too rare or too common.
\end{abstract}

Keywords: dormancy, quiescence, Lyapunov exponent, Floquet multipliers, Random Perron-Frobenius Theorem, fitness.

\footnotetext{
*Supported by NSF Grant DMS 0414270, Department of Mathematics, Arizona State University, Tempe, AZ
} 


\section{Introduction}

The ability of certain bacterial cells to reversibly enter a dormant (quiescent, non-viable, persister) state has important implications for their survival particularly in environments involving stress in the form of extreme temperatures, water scarcity, nutrient scarcity, or the presence of antibiotics [12]. Dormancy refers to a reversible state, which may persist for extended periods, in which cells exhibit low levels of metabolic activity and do not divide. They also are more tolerant of environmental stresses than active cells. The review articles by Kaprelyants et al. [12, 13] are particularly useful. They note that "the mechanism(s) of the transition of active cells into dormant cells are as yet little understood" and cite internal factors such as DNA damage, or loss of components such as ribosomes or transcription factors that may play a role as may external factors such as key resources falling below a critical threshold.

Bacterial dormancy plays a significant role in disease and human health in two ways. First, dormant pathogens may produce latent infections when activated (e.g., TB and cystic fibrosis) and second, dormant bacteria do not respond to many antimicrobial agents because these agents are typically only effective against growing cells. This resistance to antibiotics by a small subset of a bacterial population has long been observed. Balaban et. al. [3] observe that this resistance does not have a genetic origin because these "persister" cells, as the resistant cells are termed, can later regrow an antibiotic-sensitive population of "normal" cells.

There is a developing modeling literature seeking to understand when dormancy is a favorable strategy. Much of it, like our own earlier work [14], see also the references therein, is set in a time-homogeneous environment where dormancy would not be expected to be a competitive strategy. However, there is a growing body of work in which the environment is assumed to be time varying. Bar et. al. [4] develop a strategic model to highlight the survival value of a dormancy stage in bacteria which are subjected to frequent environmental stress conditions such as low soil humidity. Balaban et. al. [3] develop a simple two-component model consisting of persisters and normal cells to compare with their data on the relative size of the persister subpopulation of $E$. coli before and after antibiotic treatment. Building on this work, Kussell et. al. [16] extend the model of Balaban by including periodic antibiotic treatment. They focus on the fitness of the population determined by the dominant Lyapunov exponent. They also use numerical 
simulations to explore random environments. This work is extended and generalized to multiple bacterial strategies in a random environment which jumps between the finite set of environmental states in a beautiful paper by Kussell and Leibler [17]. They distinguish between two different bacterial strategies: stochastic and responsive. In the former, bacteria transit between different strategies at rates that are independent of the environmental state; in the latter, bacteria sense the environmental state and respond by switching to the optimal strategy for that environment at a constant rate. They compare the fitness of different strategists assuming that the duration of the environmental states is long enough that an equilibrium value of the state vector is attained before switching to a new environment. They also consider optimal transit rates.

Our focus in this work, building on our earlier results [14] as well as on the work of Kussell and Leibler [17], is on quiescence as a response to starvation but the mathematics is the same as for persister cells in response to antibiotic stress. The questions we seek to answer include: when is the capability of dormancy advantageous for an organism? Under what circumstances does it raise fitness? Which strategies for transiting between dormant and active states are favored?

\section{A Model of Dormancy in Time-Varying En- vironment}

As it is not easy to answer the questions posed above in any general setting, we seek to simplify as much as possible in our search for answers. Think of a bacterium that requires resources to survive. Resources are supplied at unpredictable times and in unpredictable amounts. We assume bacteria have a resource threshold $r_{0}$ such that the specific growth rate is $m>0$ if resource levels $r$ exceed $r_{0}$ and is zero if $r$ is below $r_{0}$. We suppose our population faces a constant specific death rate, independent of the environment, but that a quiescence-capable (dormancy-capable) organism can lower its death rate by entering the dormant state. A simple mathematical model of such an organism would feature a time-dependent specific reproduction rate $f(t)$ which takes only two values 0 and $m$; to this organism, life looks like a series 


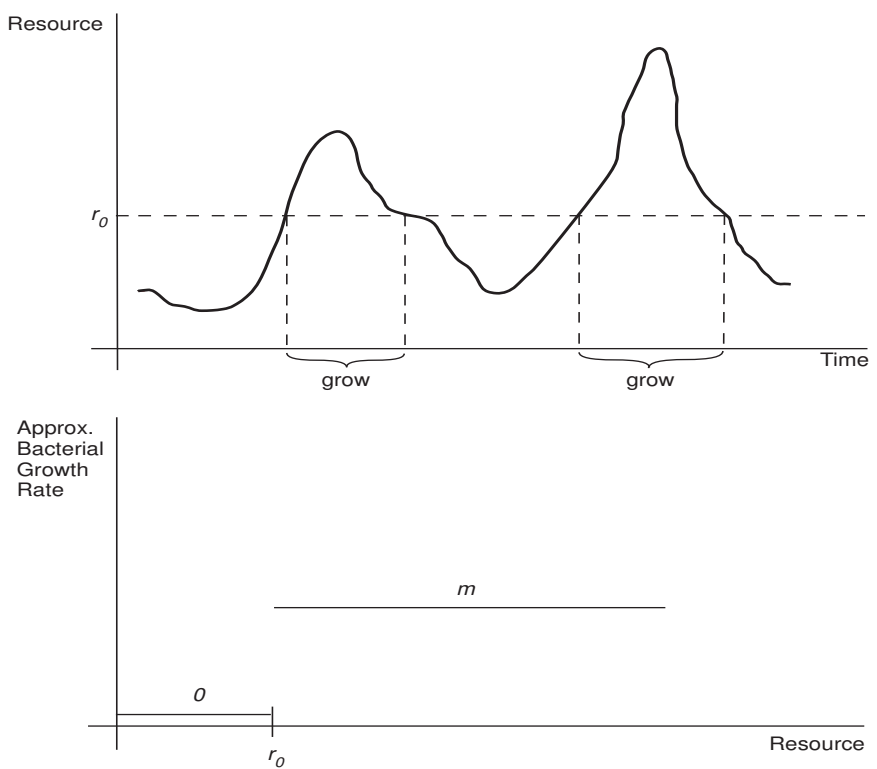

Figure 1: Bacterial growth in a "boom and bust" environment.

of "good times" where $f=m$ and "bad times" when $f=0$ :

$$
f(t)=\left\{\begin{array}{cc}
m, & \text { good times } \\
0, & \text { bad times }
\end{array}\right.
$$

A dormancy-capable organism will also have the ability to transit between the dormant and active states. In the dormant state we assume it cannot reproduce but it has a lower death rate; in the active state it can reproduce but suffers a higher death rate. In summary, the net specific growth rate of an organism in the active state is $m-d_{1}$ and that of a dormant organism is $-d_{2}$ where $0<d_{2}<d_{1}$.

The dynamics of our population can be modeled as

$$
\begin{aligned}
N^{\prime} & =\left(f(t)-d_{1}-\beta(t)\right) N+\alpha(t) D \\
D^{\prime} & =\beta(t) N-\left(d_{2}+\alpha(t)\right) D
\end{aligned}
$$

where $N$ denotes active (growing) organisms and $D$ denotes dormant ones. Functions $\alpha$ and $\beta$ represent the transit rates from quiescent to active and back, respectively. These will be described below.

We will be primarily interested in the case that the future $(t \geq 0)$ consists of alternating periods of "good times" and "bad times",

$$
\text { good times } \rightarrow \text { bad times } \rightarrow \text { good times } \rightarrow \cdots
$$



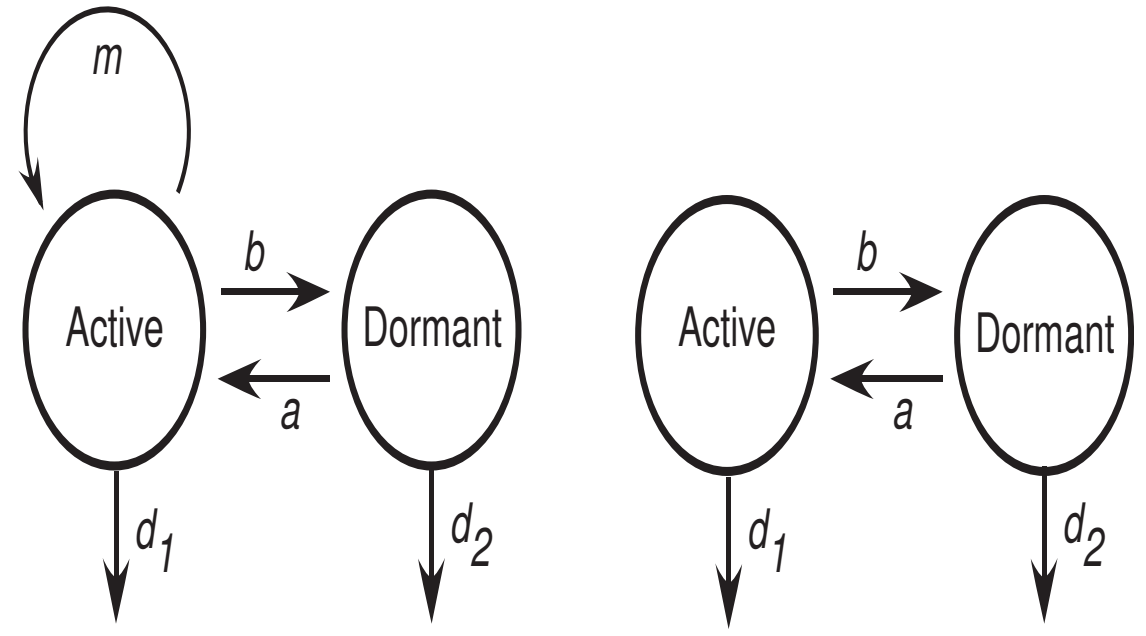

Figure 2: Stochastic switching: "good times" (left), "bad times" (right).

either of predetermined fixed lengths, giving a periodic environment, or random lengths, giving a random environment.

Bacteria may respond to their environment by transiting between dormant and active states. Again, to simplify, we follow Kussel and Leibler [16], and assume that they use one of two idealized strategies. They may completely ignore the environment and simply spend exponentially distributed times in the active and the dormant states. This is termed stochastic switching:

$$
\alpha(t)=a, \quad \beta(t)=b, \quad \text { good times and bad times }
$$

where $a, b$ are positive constants. This strategy avoids the cost of developing systems to sense the state of the environment; rather it bets on having at least some portion of the population being in the most favorable state, whatever the environmental conditions.

Alternatively, bacteria could sense their environment and begin to switch to the most advantageous state. This is responsive switching:

$$
\alpha(t)=\left\{\begin{array}{ll}
a, & \text { good times } \\
0, & \text { bad times }
\end{array} \quad \beta(t)= \begin{cases}0, & \text { good times } \\
b, & \text { bad times }\end{cases}\right.
$$

Of course, instantaneous detection of environmental stresses or bonanzas is unrealistic but we presume that bacteria respond quickly relative to the time scale of environmental changes. 

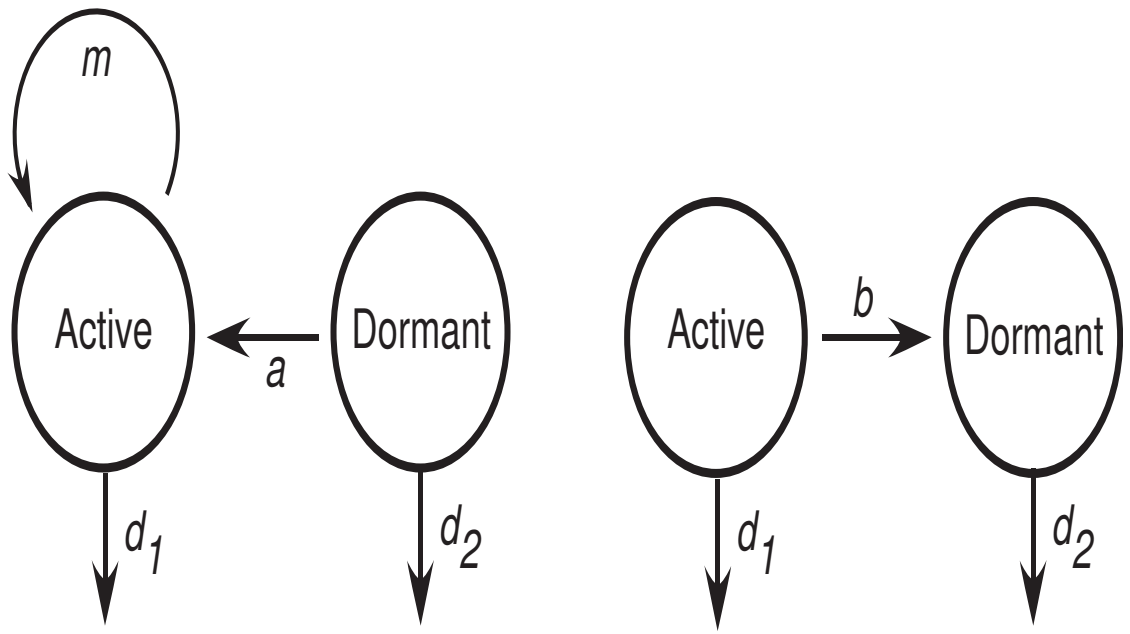

Figure 3: Responsive switching: "good times" (left), "bad times" (right).

Figures 1 and 2 depict the model flow chart in favorable and unfavorable environments for stochastic and responsive switching.

We do not assert that bacteria actually employ either of these strategies although the experimental results in [3] indicate that the stochastic switching model seems to capture type II persisters under suitable conditions. Later, a hybrid of the two idealized strategies will be described whereby organisms transit more slowly to the currently unfavorable state than the stochastic switcher does.

There would seem to be an important distinction between the stochastic and responsive strategies in so far as the transit rates $a$ and $b$ are concerned. The stochastic strategy is a population level strategy which relies on some portion of the population being favorably disposed to the current environment whatever it is. This strategy would seem to put a relatively low premium on the magnitude of transit rates. Just the opposite might be expected in the case of the responsive strategy which is an individual-based strategy. The quicker one responds, the better off the bacteria should be.

We use the term "sleepless" to denote a population whose members are incapable of entering a dormant state. Its numbers can be modeled by

$$
P^{\prime}=P\left[f(t)-d_{1}\right]
$$

Parameters $m, d_{1}, d_{2}, a, b$ will always be taken to be nonnegative. But what about their size? For bacteria we expect a doubling time of the order 
of an hour: $m-d_{1} \approx \ln (2)$. A responsive organism must be capable of responding within a generation so one expects that $a, b>m-d_{1}$; however, a stochastic transition strategy does not require transit rates on the order of a generation time. Kussell et. al. [16] take $a$ and $b$ three to six orders of magnitude smaller than $m-d_{1}$ and $d_{2}$ in their study of persister cells under periodic antibiotic stress (period in hours).

When will a quiescence-capable population do better than a sleepless population? To answer this question we need to quantify how fit each population is in the given environment. The fitness of a population is defined to be its per capita growth rate. We use the symbols $\mu_{q}^{r}$, $\mu_{q}^{s}$ for the fitness of responsive and stochastic switching, quiescence-capable populations and $\mu_{s}$ for the fitness of sleepless.

The dynamics of both populations are described by linear systems (1) and (4) respectively. For a constant environment, fitness is just the dominant eigenvalue of the coefficient matrix. In a periodic environment, fitness is precisely the largest Floquet exponent. The idea can be carried over to the random environment where the linear systems (1) and (4) are driven by a metric dynamical system [1]. However, we can also employ the more welldeveloped theory of random positive matrices [11] and the Random PerronFrobenius Theorem due to Arnold et. al. [2].

We use the manhattan norm $|x|=|(N, D)|=|N|+|D|$. If $A$ is a square matrix, we denote by $r(A)$ its spectral radius, or equivalently, the maximum modulus of an eigenvalue; its stability modulus, denoted by $s(A)$ is the maximum real part of an eigenvalue.

\section{Constant Environment Warm-up}

As a baseline, it is useful to examine the case of a constant environment first as we can obtain very explicit answers with which to compare to temporally variable environments. See [14] for a more general nonlinear system. We consider only the "good time" environment $(f=m)$ since for the "bad time" environment all fitnesses are negative resulting in extinction.

In constant "good times" a stochastically-switching quiescence-capable population is modeled by:

$$
x^{\prime}=\left(\begin{array}{cc}
m-d_{1}-b & a \\
b & -d_{2}-a
\end{array}\right) x
$$


where $x=(N, D)^{*}$ and the fitness is given by the stability modulus, $s(A)$, of the quasi-positive coefficient matrix which we denote by $A$. Its dominant eigenvalue $s(A)$ is given by

$$
\bar{\mu}_{q}^{s}=\frac{1}{2}\left(m-d_{1}-d_{2}-a-b+\sqrt{\left[m-d_{1}+d_{2}+a-b\right]^{2}+4 a b}\right)
$$

The bar on $\mu$ denotes constant environment, the superscript ' $\mathrm{s}$ ' denotes stochastic-switching strategy.

The responsive-switching population in the "good time" environment is modeled by

$$
x^{\prime}=\left(\begin{array}{cc}
m-d_{1} & a \\
0 & -d_{2}-a
\end{array}\right) x
$$

The dominant eigenvalue is

$$
\bar{\mu}_{q}^{r}=\max \left\{m-d_{1},-d_{2}-a\right\}
$$

Superscript ' $\mathrm{r}$ ' on $\mu$ indicates responsive strategy.

The sleepless population satisfies

$$
P^{\prime}=\left[m-d_{1}\right] P
$$

SO

$$
\bar{\mu}_{s}=m-d_{1}
$$

We summarize below results of simple calculations involving the fitnesses.

Proposition 1. The following hold:

1. $\bar{\mu}_{q}^{s}>0 \Leftrightarrow m-d_{1}>\frac{d_{2} b}{d_{2}+a} ; \bar{\mu}_{s}, \bar{\mu}_{q}^{r}>0 \Leftrightarrow m-d_{1}>0$.

2. $\min \left\{m-d_{1},-d_{2}\right\} \leq \bar{\mu}_{q}^{s} \leq \max \left\{m-d_{1},-d_{2}\right\}$.

3. $\bar{\mu}_{q}^{s}, \bar{\mu}_{q}^{r}$ are monotone increasing in $m$, decreasing in $d_{i}, i=1,2$.

4. $\bar{\mu}_{q}^{s}<\bar{\mu}_{s}$ if $m-d_{1}>-d_{2} ; \bar{\mu}_{q}^{s} \geq \bar{\mu}_{s}$ if $m-d_{1} \leq-d_{2}$.

5. $\bar{\mu}_{s} \leq \bar{\mu}_{q}^{r}$ and equality holds when $m-d_{1} \geq-d_{2}-a$.

6. $\bar{\mu}_{q}^{s}$ is increasing in a and decreasing in $b$ when $m-d_{1}>-d_{2}$. 
The formula for $\bar{\mu}_{q}^{s}$ is not convenient for considering what happens as $a, b$ get very large (the so-called fast switching limit). See Hadeler and Hillen [10] for a more general approach.

Proposition 2. [Fast Switching Limits] If $a \rightarrow \infty$ and $b \rightarrow \infty$ such that $a /(a+b) \rightarrow \rho$ and $b /(a+b) \rightarrow 1-\rho$, then $\bar{\mu}_{q}^{s} \rightarrow\left(m-d_{1}\right) \rho-d_{2}(1-\rho)$.

$\bar{\mu}_{q}^{r}=m-d_{1}=\bar{\mu}_{s}$ for large $a$.

Proof. The matrix $A$ has real and distinct eigenvalues for all values of $a, b>$ 0 ; they are roots of the characteristic polynomial

$$
\lambda^{2}-\left(m-d_{1}-d_{2}-a-b\right) \lambda-\left(m-d_{1}\right) d_{2}-\left(m-d_{1}\right) a+b d_{2}
$$

As the trace of $A$ goes to $-\infty$ as $a, b \rightarrow \infty$, the smaller root must go to $-\infty$ as $a, b \rightarrow \infty$ but the larger eigenvalue must remain bounded since the determinant (product of eigenvalues) is linear in $a, b$. It is easier to estimate the smaller root: divide the equation by $(a+b)^{2}$ and let $u=\lambda /(a+b)$ to obtain the quadratic satisfied by $u$. Letting $a+b \rightarrow \infty$ in the coefficients of this polynomial gives the limiting quadratic

$$
u^{2}+u=0
$$

from which we see that the smaller eigenvalue $\lambda_{1}$ of $A$ satisfies $\lambda_{1} /(a+b) \rightarrow$ -1 as $a+b \rightarrow \infty$. Since the product of the eigenvalues is the determinant, we have

$$
\lambda_{1} \lambda_{2}=-\left(m-d_{1}\right) d_{2}-\left(m-d_{1}\right) a+b d_{2}
$$

so dividing by $a+b$ and taking the limit results in $-\lambda_{2} \rightarrow-\left(m-d_{1}\right) \rho_{1}+$ $d_{2} \rho_{2}$.

In summary, comparing the fitness of sleepless with stochastic switcher and responsive switcher in an environment characterized by constant "good times" with $m-d_{1}>-d_{2}$, we have:

1. sleepless and responsive switcher have the same fitness, greater than that of stochastic switcher.

2. fitness of stochastic switcher increases with increasing $a$ but decreases with increasing $b$ while responsive switcher fitness is independent of $a, b$.

3. for stochastic switcher, any value for fitness between $m-d_{1}$ and $-d_{2}$ is achievable as a fast switching limit as $a, b \rightarrow \infty$ if $a /(a+b)$ has an appropriate limit! 


\section{Piecewise Constant Periodic Environments}

The responsive and stochastic switching strategies are depicted in the two tables below:

\begin{tabular}{|c|c|c|c||c|c|c|c|}
\hline responsive & $\alpha(t)$ & $\beta(t)$ & $\mathrm{f}(\mathrm{t})$ \\
\hline good times & $a$ & 0 & $m$ \\
\hline bad times & 0 & $b$ & 0 \\
\hline
\end{tabular} \begin{tabular}{|c|cc|c|}
\hline good times & $a$ & $b$ & $m$ \\
\hline bad times & $a$ & $b$ & 0 \\
\hline
\end{tabular}

Recall that $\alpha(t)$ is the rate of switching to active from dormant and $\beta(t)$ is the reverse rate.

The population dynamics corresponding to the two cases of stochastic switching (2) and responsive switching (3) for the quiescence-capable organism can be captured by the periodic linear system

$$
x^{\prime}=A(t) x, \quad x=(A, D)^{*}
$$

where $A(t)$ is a piecewise constant $T$-periodic matrix given by

$$
A(t)=A^{r}(t)=\left\{\begin{array}{l}
A_{1}:=\left(\begin{array}{cc}
m-d_{1} & a \\
0 & -d_{2}-a
\end{array}\right), 0<t<T_{1} \\
A_{2}:=\left(\begin{array}{cc}
-d_{1}-b & 0 \\
b & -d_{2}
\end{array}\right), T_{1}<t<T
\end{array}\right.
$$

in the case of responsive switching (3) and by

$$
A(t)=A^{s}(t)=\left\{\begin{array}{c}
A_{1}:=\left(\begin{array}{cc}
m-d_{1}-b & a \\
b & -d_{2}-a
\end{array}\right), 0<t<T_{1} \\
A_{2}:=\left(\begin{array}{cc}
-d_{1}-b & a \\
b & -d_{2}-a
\end{array}\right), T_{1}<t<T
\end{array}\right.
$$

in the case of stochastic switching (2). In either case, the fundamental matrix $\Phi(t)$, with $\Phi(0)=I$, is given by

$$
\Phi(T)=e^{A_{2}\left(T-T_{1}\right)} e^{A_{1} T_{1}} .
$$

Occasionally, we write $T_{2}=T-T_{1}$. Solutions $x(t)$ of (5) may be represented in terms of $\Phi$ by $x(t)=\Phi(t) x(0)$. After $n$ periods of good and bad times, $x(n T)=\Phi(n T) x(0)=\Phi(T)^{n} x(0)$. 
Because each $A_{i}$ has nonnegative off-diagonal entries, $e^{A_{i} t} \geq 0, t>0$ [6] and it is easily seen in both cases that $\Phi(T)>0$, meaning all entries are positive. The Perron-Frobenius Theorem ensures that $r=r(\Phi(T))>0$ is a simple eigenvalue, larger in magnitude than any other, and there is a corresponding positive eigenvector $v$ of $\Phi(T)$ and $v^{*}$ for $\Phi^{*}(T)$. We may assume $|v|=1$. The solution $x(t)$ of (5) satisfies

$$
r^{-n} x(n T) \rightarrow\left(\frac{x(0) \cdot v^{*}}{v \cdot v^{*}}\right) v
$$

See e.g. [19]. This implies that for large $n, x(n T)$ depends only on the component of the initial data $x(0)$ in the direction $v^{*}$ and that the asymptotic fractions of active and dormant bacteria are given by the components of $v$.

Here, we will be primarily interested in the asymptotic growth rate, $r$, which might be viewed as the basic reproductive number $R_{0}$, from which we define the fitness, $\tilde{\mu}_{q}$, as

$$
\tilde{\mu}_{q}=\frac{1}{T} \log r\left(e^{A_{2}\left(T-T_{1}\right)} e^{A_{1} T_{1}}\right)
$$

It is just the dominant Floquet exponent. We do not expect a nice formula for $\tilde{\mu}_{q}$ as it is notoriously difficult to compute.

We remark that the fitness would remain the same if bad times came first and good times followed since the spectral radius of $e^{A_{2}\left(T-T_{1}\right)} e^{A_{1} T_{1}}$ and of $e^{A_{1} T_{1}} e^{A_{2}\left(T-T_{1}\right)}$ are the same.

The stochastic and responsive switching strategies can be viewed as limiting cases of the hybrid strategy implicit in the periodic system

$$
x^{\prime}=A_{\lambda}(t) x, A_{\lambda}(t)=\lambda A^{r}(t)+(1-\lambda) A^{s}(t), 0 \leq \lambda \leq 1
$$

In the hybrid system, transitions between dormant and active states occur at rates which are a convex combination of the rates for the pure responsive and stochastic strategies:

$$
A_{\lambda}(t)=\left\{\begin{array}{c}
A_{1}:=\left(\begin{array}{cc}
m-d_{1}-b(1-\lambda) & a \\
b(1-\lambda) & -d_{2}-a
\end{array}\right), 0<t<T_{1} \\
A_{2}:=\left(\begin{array}{cc}
-d_{1}-b & a(1-\lambda) \\
b & -d_{2}-a(1-\lambda)
\end{array}\right), T_{1}<t<T
\end{array}\right.
$$


The difference between the hybrid and stochastic strategies is that hybrid switches more slowly than stochastic to the unfavorable state; of course, responsive does not switch at all to the unfavorable state.

The dynamics of the sleepless population are given by

$$
P^{\prime}=P\left[f(t)-d_{1}\right]=P\left\{\begin{array}{l}
m-d_{1}, 0<t<T_{1} \\
-d_{1}, T_{1}<t<T
\end{array}\right.
$$

and its fitness is given by

$$
\tilde{\mu}_{s}=m \frac{T_{1}}{T}-d_{1}
$$

We denote the fitness of a responsive-switching, quiescence-capable population by $\tilde{\mu}_{q}^{r}$ and the fitness of a stochastic-switching, quiescence-capable population by $\tilde{\mu}_{q}^{s}$. Our goal in the remainder of this section is to estimate and compare the fitnesses $\tilde{\mu}_{s}, \tilde{\mu}_{q}^{r}, \tilde{\mu}_{q}^{r}$ using both analytical and numerical methods. They depend in complicated ways on the growth, death, and transit rates and one of our goals will be to determine whether the fitnesses are monotone increasing or decreasing in some of these parameters. Our main analytical approach in dealing with the complicated non-monotone manner in which the fitnesses depend on the switching rates $a, b$ is to examine fast $(a, b \rightarrow \infty)$. We employ numerical calculations to fill in the wide gaps in our analytical results.

\subsection{Responsive switching}

Tedious computations show that $\Phi(T)=e^{\left(m-d_{1}+d_{2}\right) T_{1}-d_{2} T} Z$ where $Z$ has columns given by:

$$
\left(\begin{array}{c}
e^{-\left(d_{1}-d_{2}+b\right) T_{2}} \\
\frac{b}{d_{1}-d_{2}+b}\left[1-e^{-\left(d_{1}-d_{2}+b\right) T_{2}}\right]
\end{array}\right)
$$

and

$$
\left\{\begin{array}{c}
\left(\begin{array}{c}
\frac{a}{m-d_{1}+d_{2}+a}\left[1-e^{-\left(m-d_{1}+d_{2}+a\right) T_{1}}\right] e^{-\left(d_{1}-d_{2}+b\right) T_{2}} \\
e^{-\left(m-d_{1}+d_{2}+a\right) T_{1}}+\frac{a b\left[1-e^{-\left(m-d_{1}+d_{2}+a\right) T_{1}}\right]\left[1-e^{\left.-\left(d_{1}-d_{2}+b\right) T_{2}\right]}\right.}{\left(m-d_{1}+d_{2}+a\right)\left(d_{1}-d_{2}+b\right)}
\end{array}\right), \\
m-d_{1}+d_{2}+a \neq 0 \\
\left(\begin{array}{c}
a T_{1} e^{-\left(d_{1}-d_{2}+b\right) T_{2}} \\
e^{-\left(m-d_{1}+d_{2}+a\right) T_{1}}\left(1+\frac{a b T_{1}}{d_{1}-d_{2}+b}\left[1-e^{-\left(d_{1}-d_{2}+b\right) T_{2}}\right]\right)
\end{array}\right), \\
m-d_{1}+d_{2}+a=0
\end{array}\right.
$$


The determinant is given by $\operatorname{det} \Phi(T)=e^{(m-a+b) T_{1}-\left(d_{1}+d_{2}+b\right) T}$. Recall that the cycle time $T$ is the sum of good times $T_{1}$ followed by bad times $T_{2}$ and that the death rate for dormant bacteria is smaller than for active bacteria: $d_{2}<d_{1}$.

We denote by $\tilde{\mu}_{q}^{r}$ the fitness of a responsive-switching quiescence-capable population in periodic environment. It has the expected monotonicity properties described below.

Proposition 3. $\tilde{\mu}_{q}^{r}$ satisfies

(a) $\tilde{\mu}_{q}^{r}$ is increasing in $m$.

(b) $\tilde{\mu}_{q}^{r}$ is decreasing in $d_{i}, i=1,2$, and in $T$ if $T>\frac{\ln \left(d_{1}+b\right)-\ln \left(d_{2}\right)}{d_{1}+b-d_{2}}$.

(c) $\tilde{\mu}_{q}^{r} \rightarrow-d_{2}, T_{1} \searrow 0 ; \tilde{\mu}_{q}^{r} \rightarrow \max \left\{m-d_{1},-d_{2}-a\right\}, T_{2} \searrow 0$

Proof. Recall that the spectral radius is monotone increasing in any entry of a positive matrix $[5,20]$. This together with some computation leads to the monotonicity assertions with respect to $m$ and $d_{i}$ in (a) and (b).

Consider the dependence on $T$. The top row entries of $\Phi(T)$ are clearly decreasing in $T$. The bottom row entries contain the factor $e^{-c T}-e^{-k T}$ where $c=d_{1}+b, k=d_{2}$ so $0<c<k$. It is easily checked that this function of $T$ has a negative derivative if $T>\frac{\ln (k / c)}{k-c}$.

Part (c) follows from continuity of the spectral radius so $\tilde{\mu}_{q}^{r} \rightarrow s\left(A_{2}\right), T_{1} \rightarrow$ 0 , where $s\left(A_{2}\right)$ denotes the stability modulus of matrix $A_{2}$. The entry $Z_{12}$ of $Z$ satisfies $Z_{21} \rightarrow 0$ as $T_{2} \rightarrow 0$ leading to the final assertion.

The spectral radius of the positive matrix $\Phi(T), e^{\tilde{\mu}_{q}^{r} T}$, must lie between its column sums $[5,20]$. These are given by

$$
c_{1}=e^{\left(m-d_{1}+d_{2}\right) T_{1}-d_{2} T} q, \quad c_{2}=e^{\left(m-d_{1}+d_{2}\right) T_{1}-d_{2} T} p
$$

where $p, q$ are given by

$$
\begin{aligned}
& q=e^{-\left(d_{1}-d_{2}+b\right) T_{2}}+\frac{b\left[1-e^{-\left(d_{1}-d_{2}+b\right) T_{2}}\right]}{d_{1}-d_{2}+b} \in(0,1) \\
& p=e^{-\left(m-d_{1}+d_{2}+a\right) T_{1}}+q \frac{a\left[1-e^{-\left(m-d_{1}+d_{2}+a\right) T_{1}}\right]}{\left(m-d_{1}+d_{2}+a\right)}
\end{aligned}
$$


Observe that $p \in(0,1)$ if $m-d_{1}+d_{2}+a>0$. Also note that $q$ depends on $b$ while $p$ depends on $a, b$ and both approach 1 as $a, b \rightarrow \infty$.

These arguments lead to very crude estimates and the existence of a unique fast-switching limit fitness.

Proposition 4. If $m-d_{1}+d_{2}+a>0$, the following estimate holds:

$$
\frac{b}{b+d_{1}-d_{2}} \frac{a}{a+m-d_{1}+d_{2}} e^{\left(m-d_{1}\right) T_{1}-d_{2} T_{2}} \leq e^{\tilde{\mu}_{q}^{r} T}<e^{\left(m-d_{1}\right) T_{1}-d_{2} T_{2}}
$$

In particular, $\tilde{\mu}_{q}^{r}<0$ if $\left(m-d_{1}+d_{2}\right) \frac{T_{1}}{T}<d_{2}$.

Moreover, in the limit as $a, b \rightarrow \infty$, we have

$$
\lim _{a, b \rightarrow \infty} \tilde{\mu}_{q}^{r}=\left(m-d_{1}+d_{2}\right) \frac{T_{1}}{T}-d_{2} .
$$

Proof. As noted above, $e^{\tilde{\mu}_{q}^{r} T}=e^{\left(m-d_{1}+d_{2}\right) T_{1}-d_{2} T} r$ where $r$ lies between $p$ and $q$ and $\frac{b}{b+d_{1}-d_{2}} \leq q<1$ and $q \frac{a}{a+m-d_{1}+d_{2}} \leq p<1$.

The following result shows that in environments characterized by brief good times followed by long stress durations, the quiescence-capable population is more fit.

Proposition 5. [Responsive beats sleepless if good times are brief]

$$
\frac{T_{1}}{T}<\frac{d_{1}-d_{2}}{m+a} \Rightarrow \tilde{\mu}_{q}^{r}>\tilde{\mu}_{s}
$$

Proof. We examine $\mu:=\tilde{\mu}_{q}^{r}-\tilde{\mu}_{s}$, where $e^{\mu T}$ may be viewed as the spectral radius of $\Psi(T):=e^{-\mu_{s} T} \Phi(T)$. Now

$$
\begin{aligned}
\operatorname{det} \Psi(T) & \left.=\operatorname{det} e^{-\mu_{s} T} \Phi(T)\right)=e^{-2 \mu_{s} T} \operatorname{det} \Phi(T)=e^{\left(d_{1}-d_{2}\right) T-(m+a) T_{1}-b T_{2}} \\
\operatorname{tr} \Psi(T) & =\operatorname{tr}\left(e^{-\mu_{s} T} \Phi\right)=e^{-\mu_{s} T} \operatorname{tr} \Phi(T) \\
& =e^{\left(d_{1}-d_{2}\right) T-(m+a) T_{1}}+e^{-b T_{2}} \\
& +T_{1} \frac{a b}{d_{1}-d_{2}+b} e^{-(m+a) T_{1}} \frac{e^{(m+a) T_{1}}-e^{\left(d_{1}-d_{2}\right) T_{1}}}{(m+a) T_{1}-\left(d_{1}-d_{2}\right) T_{1}}\left(e^{\left(d_{1}-d_{2}\right) T_{2}}-e^{-b T_{2}}\right)
\end{aligned}
$$

We observe that

$$
\operatorname{tr} \Psi(T)-\operatorname{det} \Psi(T)>e^{\left(d_{1}-d_{2}\right) T-(m+a) T_{1}}+e^{-b T_{2}}-e^{\left(d_{1}-d_{2}\right) T-(m+a) T_{1}-b T_{2}}
$$


SO

$$
\begin{aligned}
\frac{T_{1}}{T} & <\frac{d_{1}-d_{2}}{m+a} \\
& \Leftrightarrow\left(d_{1}-d_{2}\right) T-(m+a) T_{1}>0 \\
& \Leftrightarrow e^{\left(d_{1}-d_{2}\right) T-(m+a) T_{1}}>1 \\
& \Leftrightarrow\left(e^{\left(d_{1}-d_{2}\right) T-(m+a) T_{1}}-1\right)\left(1-e^{-b T_{2}}\right)>0 \\
& \Leftrightarrow e^{\left(d_{1}-d_{2}\right) T-(m+a) T_{1}}+e^{-b T_{2}}-e^{\left(d_{1}-d_{2}\right) T-(m+a) T_{1}-b T_{2}}>1
\end{aligned}
$$

This gives

$$
\operatorname{tr} \Psi(T)-\operatorname{det} \Psi(T)>1
$$

showing that $\Psi(T)$ violates one of the Jury conditions [8]. This implies that the spectral radius of $\Psi(T), e^{\mu T}$ is larger than 1. Equivalently, $\mu>0$.

\subsection{Stochastic Switching}

We denote by $\tilde{\mu}_{q}^{s}$ the fitness of a stochastic-switching quiescence-capable population in periodic environment. The fundamental matrix $\Phi(t)$ is given by

$$
\Phi(T)=e^{A_{2}\left(T-T_{1}\right)} e^{A_{1} T_{1}}
$$

where

$$
A(t)=A_{T_{1}}(t)=\left\{\begin{array}{c}
A_{1}:=\left(\begin{array}{cc}
m-d_{1}-b & a \\
b & -d_{2}-a
\end{array}\right), 0<t<T_{1} \\
A_{2}:=\left(\begin{array}{cc}
-d_{1}-b & a \\
b & -d_{2}-a
\end{array}\right), T_{1}<t<T
\end{array}\right.
$$

The fitness $\tilde{\mu}_{q}^{s}$ increases with $m$ and decreases with $d_{i}$ for the same reasons cited in Proposition 3. In contrast to the responsive case, here we can show that it is also increasing in $T_{1}$.

Lemma 1. The map $T_{1} \rightarrow \tilde{\mu}_{q}^{s}\left(T_{1}\right)$ is continuous and increasing on $[0, T]$ and

$$
s\left(A_{2}\right) \leq \tilde{\mu}_{q}^{s}\left(T_{1}\right) \leq s\left(A_{1}\right)=\bar{\mu}_{q}
$$

where $s\left(A_{i}\right)$ is the stability modulus of $A_{i}$. 
Proof. Since $A_{1} \geq A_{2}$ and $A_{1} \neq A_{2}$ we may conclude that $e^{A_{1} t} \geq e^{A_{2} t}>0$ and $e^{A_{1} t} \neq e^{A_{2} t}$ for $t>0$. Therefore, if $0 \leq T_{1}<T_{1}^{\prime} \leq T$, we have

$e^{A_{2}\left(T-T_{1}\right)} e^{A_{1} T_{1}}=e^{A_{2}\left(T-T_{1}^{\prime}+T_{1}^{\prime}-T_{1}\right)} e^{A_{1} T_{1}} \leq e^{A_{2}\left(T-T_{1}^{\prime}\right)} e^{A_{1}\left(T_{1}^{\prime}-T_{1}\right)} e^{A_{1} T_{1}}=e^{A_{2}\left(T-T_{1}^{\prime}\right)} e^{A_{1} T_{1}^{\prime}}$

Moreover, equality does not hold! It follows from the Perron-Frobenius theory [5] that $T_{1} \rightarrow r\left(T_{1}\right) \equiv r\left(e^{A_{2}\left(T-T_{1}\right)} e^{A_{1} T_{1}}\right)$ is strictly increasing for $T_{1} \in[0, T]$ and

$$
r(0)=r\left(e^{A_{2} T}\right)=e^{s\left(A_{2}\right) T} \leq r\left(T_{1}\right) \leq e^{s\left(A_{1}\right) T}=r\left(e^{A_{1} T}\right)=r(T)
$$

where $s(A)$ is the stability modulus (largest real eigenvalue) of $A$. Since $\tilde{\mu}_{q}^{s}=\tilde{\mu}_{q}^{s}\left(T_{1}\right)=\left(\log r\left(T_{1}\right)\right) / T$ we see that $T_{1} \rightarrow \tilde{\mu}_{q}^{s}\left(T_{1}\right)$ is strictly increasing and (10) holds.

Continuity follows since $A \rightarrow r(A)$ is continuous and $T_{1} \rightarrow e^{A_{2}\left(T-T_{1}\right)} e^{A_{1} T_{1}}$ is continuous.

If either good times are rare or bad times are rare, then responsive is more fit than stochastic.

Proposition 6. [responsive better than stochastic when good times are rare or bad times are rare]

$$
\tilde{\mu}_{q}^{r}>\tilde{\mu}_{q}^{s}, \quad T_{1} \approx 0
$$

If $m-d_{1}>-d_{2}$ then

$$
\tilde{\mu}_{q}^{r}>\tilde{\mu}_{q}^{s}, \quad T-T_{1} \approx 0
$$

Proof. By continuity of the maps $T_{1} \rightarrow \tilde{\mu}_{q}^{s}\left(T_{1}\right)$ and $T_{1} \rightarrow \tilde{\mu}_{q}^{r}\left(T_{1}\right)$ for $0 \leq T_{1} \leq$ $T$, it suffices to show that $\tilde{\mu}_{q}^{s}\left(T_{1}=0\right)<\tilde{\mu}_{q}^{r}\left(T_{1}=0\right)$, or equivalently, that

$$
s\left(\begin{array}{cc}
-d_{1}-b & a \\
b & -a-d_{2}
\end{array}\right)<s\left(\begin{array}{cc}
-d_{1}-b & 0 \\
b & -d_{2}
\end{array}\right)
$$

Since the dominant eigenvalue of the matrix on the right is $-d_{2}$, we just need to show that

$$
s\left(\begin{array}{cc}
-d_{1}-b+d_{2} & a \\
b & -a
\end{array}\right)<0
$$

As the trace of this matrix is negative and its determinant $a\left(d_{1}-d_{2}\right)>0$, we are done.

Proposition 1, case (5), and similar reasoning implies the second assertion. 
Similar reasoning can be used to compare fitness of sleepless and the stochastic switcher. Stochastic switching is better than being sleepless when good times are rare.

Proposition 7. If $m-d_{1}+d_{2}>0$, then there exists $T_{1}^{*} \in(0, T)$ such that

$$
\tilde{\mu}_{q}^{s}>\tilde{\mu}_{s} \Leftrightarrow T_{1}<T_{1}^{*}
$$

Proof. $\tilde{\mu}_{q}^{s}>\tilde{\mu}_{s}$ if and only if $r\left(e^{A_{2} T_{2}} e^{A_{1} T_{1}}\right)>e^{m T_{1}-d_{1} T}$ if and only if

$$
r\left(e^{d_{1} T_{2}} e^{A_{2} T_{2}} e^{-\left(m-d_{1}\right) T_{1}} e^{A_{1} T_{1}}\right)>1
$$

if and only if $r\left(e^{C_{2} T_{2}} e^{C_{1} T_{1}}\right)>1$ where $C_{1}=A_{1}-\left(m-d_{1}\right) I$ and $C_{2}=A_{2}+d_{1} I$. As $C_{2}>C_{1}$, an argument just like in Lemma 1 shows that the map $T_{1} \rightarrow$ $r\left(T_{1}\right) \equiv r\left(e^{C_{2} T_{2}} e^{C_{1} T_{1}}\right)$ is strictly decreasing. $r(0)=r\left(e^{C_{2} T}\right)=e^{s\left(C_{2}\right) T}>1$ since $s\left(C_{2}\right)>0\left(\operatorname{det} C_{2}<0\right) \cdot r(T)=e^{C_{1} T}=e^{s\left(C_{1}\right) T}<1$ since the trace of $C_{1}$ is negative and its determinant is positive if $m-d_{1}+d_{2}>0$. It follows that there is a unique $T_{1}^{*}$ such that $r\left(T_{1}^{*}\right)=1$.

Just as for the constant environment, there is an interval of potential limiting fitnesses that can be attained in the limit $a, b \rightarrow \infty$.

Proposition 8. If $a, b \rightarrow \infty$ such that $\frac{a}{a+b}=q, \frac{b}{a+b}=p$, then

$$
\tilde{\mu}_{q}^{s} \rightarrow\left[m \frac{T_{1}}{T}-d_{1}\right] q-d_{2} p
$$

Proof. We change variables in (5) following [10]. Let $u=N+D$ (total population) and $v=b N-a D$ (net flux) and $\epsilon=1 /(a+b)$. Tedious calculations give the equations:

$$
\begin{aligned}
u^{\prime} & =r u+\epsilon c v \\
\epsilon v^{\prime} & =-v+\epsilon s v+f u
\end{aligned}
$$

where $r=\left(m-d_{1}\right) q-d_{2} p, c=m-d_{1}+d_{2}, s=p\left(m-d_{1}\right)-q d_{2}, f=$ $p q\left(m-d_{1}+d_{2}\right)$. We write $B_{1}$ for the coefficient matrix associated with (12). The solution with initial data $(1,0)^{*}$ is easily estimated using standard singular perturbation theory as

$$
(u, v)\left(T_{1}\right)=e^{r T_{1}}(1, f)+O(\epsilon), \epsilon \rightarrow 0 .
$$


Indeed, for small $\epsilon>0, v$ rapidly equilibrates to $f u(0)$ while $u$ remains near $u(0)=1$; then $u$ and $v$ remain in proportion $v=f u$ while $u$ changes according to $u^{\prime}=r u$. See [9].

Similarly, the solution corresponding to initial data $(0,1)^{*}$ is

$$
(u, v)\left(T_{1}\right)=(0,0)+O(\epsilon), \epsilon \rightarrow 0
$$

So in the new coordinates

$$
e^{B_{1} T_{1}}=e^{r T_{1}}\left(\begin{array}{ll}
1 & 0 \\
f & 0
\end{array}\right)+O(\epsilon)
$$

In these same coordinates the coefficient matrix $B_{2}$, similar to matrix $A_{2}$, has $m=0$ (note this changes $r, c, s, f$ to $r^{\prime}, c^{\prime}, s^{\prime}, f^{\prime}$ ). Therefore,

$$
e^{B_{2} T_{2}}=e^{r^{\prime} T_{2}}\left(\begin{array}{cc}
1 & 0 \\
f^{\prime} & 0
\end{array}\right)+O(\epsilon)
$$

Multiplying these two gives a matrix similar to $e^{A_{2} T_{2}} e^{A_{1} T_{1}}$, namely

$$
e^{B_{2} T_{2}} e^{B_{1} T_{1}}=e^{r T_{1}} e^{r^{\prime} T_{2}}\left(\begin{array}{cc}
1 & 0 \\
f^{\prime} & 0
\end{array}\right)+O(\epsilon)
$$

so by continuity of the spectrum

$$
r\left(e^{A_{2} T_{2}} e^{A_{1} T_{1}}\right)=e^{r^{\prime}\left(T-T_{1}\right)} e^{r T_{1}}+O(\epsilon),
$$

which implies (11).

Remark 1. Comparing the fast switching limits of responsive and stochastic, one can see that if $m-d_{1}+d_{2}>0$ then the limiting fitness of responsive switching exceeds the limiting fitness of stochastic switching as $a, b \rightarrow \infty$. The limiting responsive fitness also exceeds the fitness of sleepless. The limiting fitness of stochastic switching exceeds the fitness of sleepless precisely when $m \frac{T_{1}}{T}<d_{1}-d_{2}$.

We present some numerical comparisons of the fitness $\left(R_{0}=e^{\mu T}\right)$ for sleepless, responsive switcher, and a stochastic switcher as a function of various parameters in the following figures. All our calculations were performed using matlab to calculate the exponential matrices $e^{A_{i} t}, i=1,2$, column by column using standard ODE solvers. The matrices were then multiplied and 
the dominant eigenvalue of the product computed. In all of figures we let the period of the environmental forcing be $T=10$ and used the following parameter values: $m=2, d_{1}=0.1, d_{2}=0.01$.

Figure 4 provides comparisons of the fitness $\left(R_{0}=e^{\mu T}\right)$ for sleepless, responsive switcher, and a stochastic switcher as a function of the length of good times $T_{1}$. A notable feature is that stochastic switching can be more fit than responsive switching in intermediate ranges of $T_{1}$. This is consistent with Proposition6 which shows that responsive switching is more fit when $T_{1} \approx 0$ and when $T_{1} \approx T$.
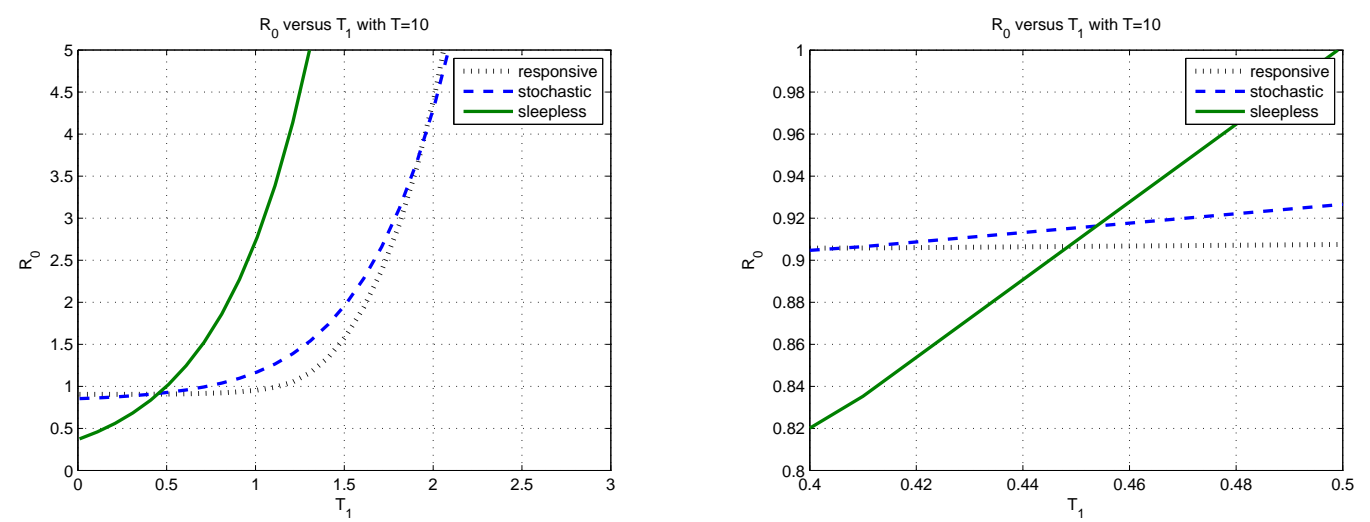

Figure 4: $R_{0}$ versus $T_{1}$ with $a=0.02, b=0.2$. Right figure is a blow-up of the intersection.

Figure 5 provides comparisons of the fitness as a function of the transit rates $a, b$ with the length of good times fixed at $T_{1}=1$. We increase $a$ and $b$ simultaneously, keeping a constant ratio of $b / a=10$. Remarkably, the fitnesses are not monotone with respect to the parameters $a$ and $b$ when increased in tandem when transit rates are small.

Observe that as $a, b \rightarrow 0$

$$
\tilde{\mu}_{q}^{r}, \tilde{\mu}_{q}^{s} \rightarrow \max \left\{m \frac{T_{1}}{T}-d_{1},-d_{2}\right\}
$$

so the fitnesses agree with that of sleepless when $m \frac{T_{1}}{T}-d_{1}>-d_{2}$ in the limit $a, b \rightarrow 0$. This explains why the curves come together in Figure 5 and 6 . Figure 6 is the same as Figure 5 except the ratio $b / a=1$.

Figure 7 gives the strategy with highest fitness for a range of values of $b$ and $T_{1}$ where $a / b=0.1$ and the other parameters are as above. Key 
points are that each strategy is dominant in some open set of values of the two parameters, responsive is dominant for small $T_{1}$ and all $b$, stochastic is dominant in a narrow region of moderate $T_{1}$ and small $b$, sleepless dominates at large values of $T_{1}$.
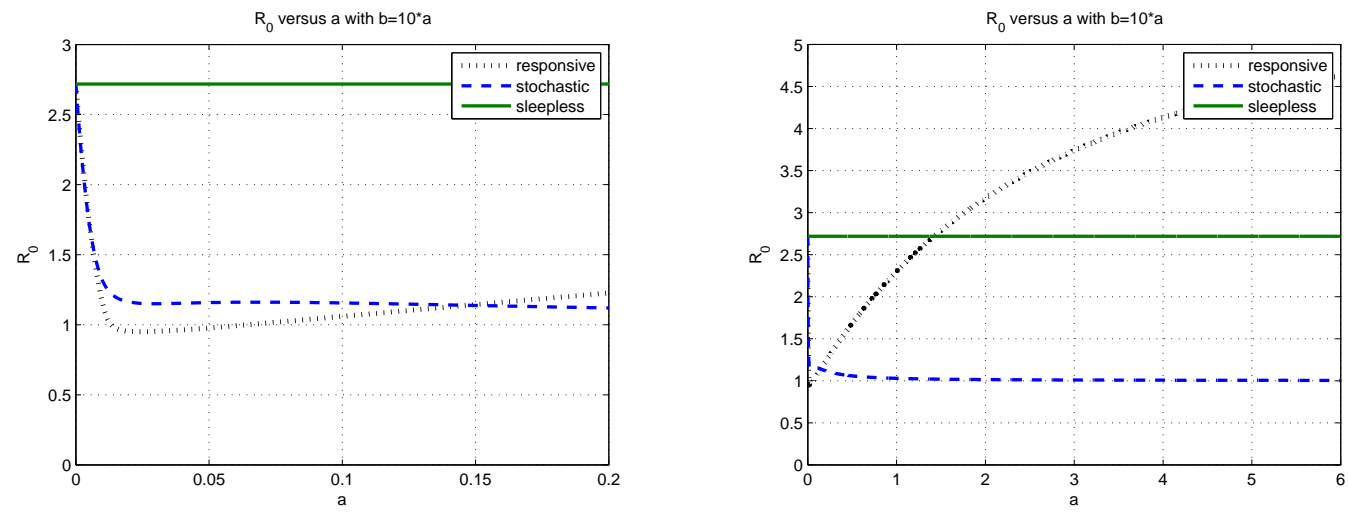

Figure 5: $R_{0}$ versus $a$ with $b=10 * a$; small $a$ (left) and large $a$ (right)
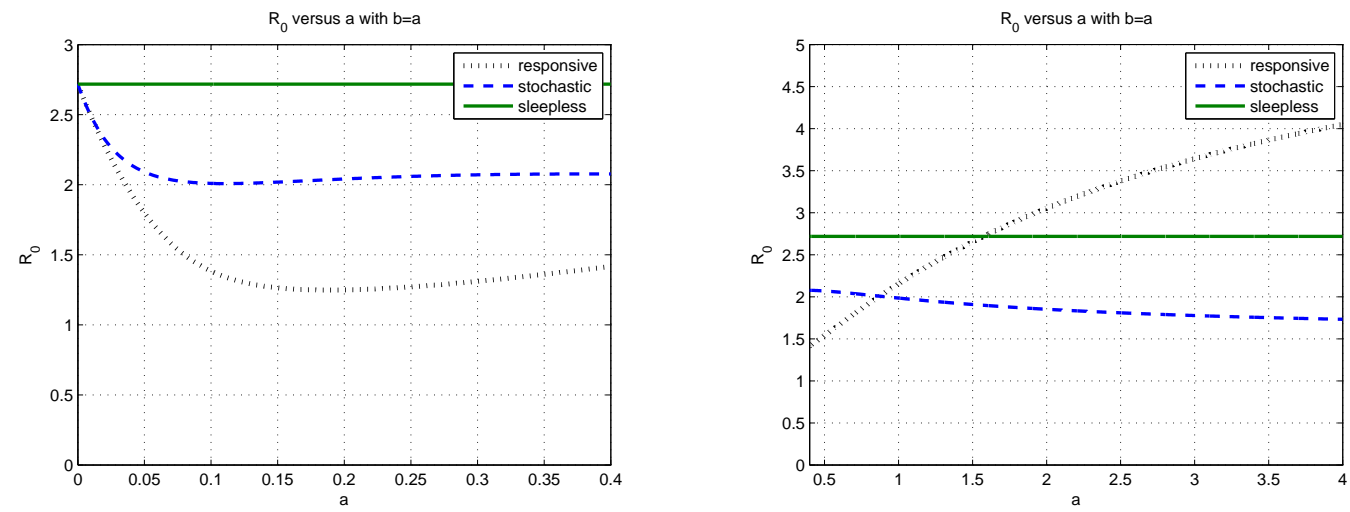

Figure 6: $R_{0}$ versus $a$ with $b=a$; small $a$ (left), large $a$ (right)

\section{Random Environment}

We consider an environment in which conditions switch repeatedly from stress to growth and from growth to stress at random times denoted by 


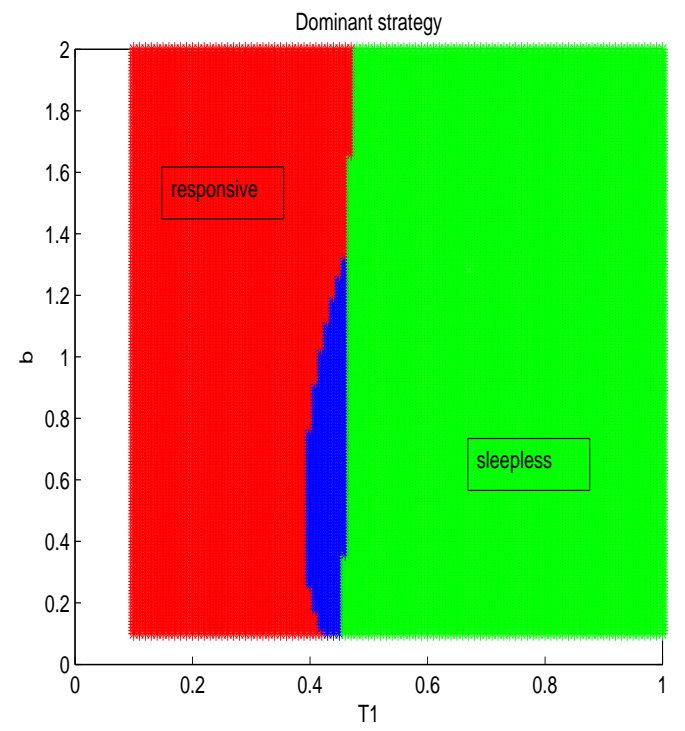

Figure 7: Dominant strategy as a function of $T_{1}$ and $b$ with $a / b=0.1$. Stochastic is dominant in the black (blue in color) region.

$T_{i}$ and $\tau_{i},(i \in \mathbb{Z})$ respectively:

$T_{1}$ units good times $\rightarrow \tau_{1}$ units bad times $\rightarrow T_{2}$ units good times $\rightarrow \cdots$

$T_{i}\left(\tau_{i}\right)(i \in \mathbb{Z})$ are independent and identically distributed random variables with corresponding distribution function $F^{T}\left(F^{\tau}\right)$. Let $E T_{i}=T$ and $E \tau_{i}=\tau$ denote the expected values of good and bad times, respectively. As we are primarily interested in the dominant Lyapunov exponent which measures the exponential rate of growth, it will not matter whether we begin with good times or with bad times or, more generally, if we start at some arbitrary time.

The sleepless population satisfies

$$
P^{\prime}=P\left[f(t)-d_{1}\right]
$$

Its fitness is given by

$$
\hat{\mu}_{s}=m \frac{\tau}{T+\tau}-d_{1}
$$


Indeed, with $P(0)=1$, following $n$ good times we have

$$
\begin{aligned}
P(t) & =\exp \int_{0}^{t}\left(f(s)-d_{1}\right) d s \\
& \left.=\exp \left(-d_{1} t\right) \exp \left[m \sum_{i=1}^{n} T_{i}\right)\right], \sum_{i=1}^{n-1}\left(T_{i}+\tau_{i}\right)+T_{n}<t \leq \sum_{i=1}^{n}\left(T_{i}+\tau_{i}\right)
\end{aligned}
$$

If $t_{n}=\sum_{i=1}^{n-1}\left(T_{i}+\tau_{i}\right)+T_{n}$ denotes the end time of the $n$-th "good time", the fitness can be computed as

$$
\begin{aligned}
\mu_{s} & =\lim _{n \rightarrow \infty} \frac{1}{t_{n}} \log P\left(t_{n}\right) \\
& =\lim _{n \rightarrow \infty} \frac{1}{t_{n}}\left(-d_{1} t_{n}+m \sum_{i=1}^{n} T_{i}\right) \\
& =-d_{1}+m \lim _{n \rightarrow \infty} \frac{1}{t_{n}} \sum_{i=1}^{n} T_{i} \\
& =-d_{1}+m \lim _{n \rightarrow \infty} \frac{\frac{1}{n} \sum_{i=1}^{n} T_{i}}{\frac{1}{n} \sum_{i=1}^{n} T_{i}+\frac{1}{n} \sum_{i=1}^{n-1} \tau_{i}} \\
& =-d_{1}+m \frac{T}{T+\tau}
\end{aligned}
$$

where we have used the strong law of large numbers [7].

The dynamics of a quiescence-capable population are given by

$$
x^{\prime}=A(t) x
$$

where $x$ is the population vector $(N, D)^{*}$ and $A(t)$ takes the value $A_{1}$ in good times and $A_{2}$ in bad times. Here, we consider the responsive and stochastic case simultaneously.

After one cycle of good and bad times, the fundamental matrix is given by

$$
x\left(T_{1}+\tau_{1}\right)=e^{A_{2} \tau_{1}} e^{A_{1} T_{1}} x(0)
$$

Define

$$
M\left(T_{i}, \tau_{i}\right):=e^{A_{2} \tau_{i}} e^{A_{1} T_{i}}
$$

Then at the end of the $n$-th stress-growth interval, we have

$$
x\left(\sum_{i=1}^{n} T_{i}+\tau_{i}\right)=M\left(T_{n}, \tau_{n}\right) M\left(T_{n-1}, \tau_{n-1}\right) \ldots M\left(T_{1}, \tau_{1}\right) x(0)
$$


which is a product of positive random matrices. Fitness is defined as the dominant Lyapunov exponent, denoted by $\hat{\mu}_{q}$. We must show that this is well-defined.

Proposition 9. Fitness, defined as the dominant Lyapunov exponent, is well-defined. It is given by $\hat{\mu}_{q}=\lambda /(T+\tau)$ where

$$
\lambda=\lim _{n \rightarrow \infty} \frac{1}{n} \log \left|M\left(T_{n}, \tau_{n}\right) M\left(T_{n-1}, \tau_{n-1}\right) \ldots M\left(T_{1}, \tau_{1}\right) x\right|, x>0
$$

is the Perron-Frobenius eigenvalue guaranteed by Random Perron-Frobenius Theorem of [2]. In particular, $\lambda$ is independent of $x>0$.

Proof. We compute:

$$
\begin{aligned}
\hat{\mu}_{q} & =\lim _{n \rightarrow \infty} \frac{1}{\sum_{i=1}^{n}\left(T_{i}+\tau_{i}\right)} \log \left|x\left(\sum_{i=1}^{n}\left(T_{i}+\tau_{i}\right)\right)\right| \\
& =\lim _{n \rightarrow \infty} \frac{n}{\sum_{i=1}^{n}\left(T_{i}+\tau_{i}\right)} \cdot \frac{1}{n} \log \left|M\left(T_{n}, \tau_{n}\right) M\left(T_{n-1}, \tau_{n-1}\right) \ldots M\left(T_{1}, \tau_{1}\right) x(0)\right| \\
& =\lim _{n \rightarrow \infty} \frac{n}{\sum_{i=1}^{n}\left(T_{i}+\tau_{i}\right)} \cdot \lim _{n \rightarrow \infty} \frac{1}{n} \log \left|M\left(T_{n}, \tau_{n}\right) M\left(T_{n-1}, \tau_{n-1}\right) \ldots M\left(T_{1}, \tau_{1}\right) x(0)\right| \\
& =\frac{1}{T+\tau} \cdot \lambda
\end{aligned}
$$

by the Random Perron-Frobenius Theorem and the strong law of large numbers. The Random Perron-Frobenius Theorem holds provided we verify that the entries of the positive matrix $M$ have the property that $\log ^{+}\left(M_{i j}(T, \tau)\right)$ and $\log ^{+}\left(1 / M_{i j}(T, \tau)\right)$ are integrable with respect to the product measure generated by distributions $F^{T}$ and $F^{\tau}$. But $\log ^{+}\left(M_{i j}(T, \tau)\right) \leq\left\|A_{2}\right\| \tau+\left\|A_{1}\right\| T$ the right hand side of which is integrable since $E(T), E(\tau)<\infty$. Here, $\|A\|$ denotes the operator norm relative to the Euclidean norm. On the other hand, it is not hard to see that each $M_{i j}(T, \tau)$ satisfies an inequality $M_{i j}(T, \tau) \geq c e^{-f T-g \tau}$ for some $c>0$ and $f, g \geq 0$ and that this implies $\log ^{+}\left(1 / M_{i j}(T, \tau)\right) \leq f T+g \tau$. Again, the right hand side is integrable.

Remark 2. The Perron-Frobenius eigenvalue $\lambda$, and therefore $\hat{\mu}_{q}$, has the expected monotonicity properties with respect to $m, d_{i}$ by virtue of its definition as a limit and by the monotonicity of the matrices $M$ in these variables.

In the stochastic switching case, we can give the same crude estimate of $\hat{\mu}_{q}^{s}$ that we gave in the periodic case for the same reasons. 
Proposition 10. In the stochastic switching case, we have

$$
s\left(A_{2}\right) \leq \hat{\mu}_{q}^{s} \leq s\left(A_{1}\right)=\bar{\mu}_{q}
$$

Proof. In the stochastic switching case, since $A_{2} \leq A_{1}$, we have $e^{A_{2}\left(T_{1}+\tau_{1}\right)} \leq$ $e^{A_{2} \tau_{1}} e^{A_{1} T_{1}} \leq e^{A_{1}\left(T_{1}+\tau_{1}\right)}$. We can compare $\lambda$ for $M\left(T_{1}, \tau_{1}\right)$ with $\lambda_{i}$ corresponding to $M_{i}\left(T_{1}, \tau_{1}\right)=e^{A_{i}\left(T_{1}+\tau_{1}\right)}$, that is $\lambda_{2} \leq \lambda \leq \lambda_{1}$. By the Random PerronFrobenius Theorem [2], $\lambda_{i}=E \log q$ where $q$ is the random variable eigenvalue and $u$ is the unit random positive eigenvector. The uniqueness of $q$ and $u$ together with the fact that $e^{A_{i}\left(T_{1}+\tau_{1}\right)} v=e^{s\left(A_{i}\right)\left(T_{1}+\tau_{1}\right)} v_{i}$ where $A_{i} v_{i}=s\left(A_{i}\right) v_{i}$, $v_{i}$ being the unique positive unit eigenvector for $A_{i}$, guarantees that $u=v_{i}$ and $q=e^{s\left(A_{i}\right)\left(T_{1}+\tau_{1}\right)}$. Therefore

$$
\lambda_{i}=E \log e^{s\left(A_{i}\right)\left(T_{1}+\tau_{1}\right)}=s\left(A_{i}\right)(T+\tau)
$$

which, together with the inequality above, gives the desired result.

A second proof uses the independence of the exponent on the initial data.

$$
\begin{aligned}
\hat{\mu}_{q}^{s} & \leq \lim _{n \rightarrow \infty} \frac{1}{\sum_{i=1}^{n}\left(T_{i}+\tau_{i}\right)} \log \left|e^{A_{1}\left(\sum_{i=1}^{n}\left(T_{i}+\tau_{i}\right)\right)} x\right| \\
& =\lim _{n \rightarrow \infty} \frac{n}{\sum_{i=1}^{n}\left(T_{i}+\tau_{i}\right)} \cdot \frac{1}{n} \log \left|e^{A_{1} \sum_{i=1}^{n}\left(T_{i}+\tau_{i}\right)} v_{1}\right| \\
& =\lim _{n \rightarrow \infty} \frac{n}{\sum_{i=1}^{n}\left(T_{i}+\tau_{i}\right)} \cdot \frac{1}{n} \log \left|e^{s\left(A_{1}\right) \sum_{i=1}^{n}\left(T_{i}+\tau_{i}\right)} v_{1}\right| \\
& =\lim _{n \rightarrow \infty} \frac{n}{\sum_{i=1}^{n}\left(T_{i}+\tau_{i}\right)} \cdot s\left(A_{1}\right)\left(\frac{1}{n} \sum_{i=1}^{n}\left(T_{i}+\tau_{i}\right)\right) \\
& =\frac{1}{(T+\tau)} \cdot s\left(A_{1}\right)(T+\tau)=s\left(A_{1}\right)
\end{aligned}
$$

In the responsive switching case, we can give the same crude estimate of $\hat{\mu}_{q}^{r}$ that we gave in the periodic case but the argument is more involved.

Proposition 11. If $m-d_{1}+d_{2}+a>0$, the following estimate holds:

$$
\frac{\log s}{T+\tau}+\left(m-d_{1}\right) \frac{T}{T+\tau}-d_{2} \frac{\tau}{T+\tau} \leq \hat{\mu}_{q}^{r} \leq\left(m-d_{1}\right) \frac{T}{T+\tau}-d_{2} \frac{\tau}{T+\tau}
$$

where $s=a b /\left(d_{1}+b-d_{2}\right)\left(d_{2}+a+m-d_{1}\right) \in(0,1)$. 
Moreover, in the limit as $a, b \rightarrow \infty$, we have

$$
\lim _{a, b \rightarrow \infty} \hat{\mu}_{q}^{r}=\left(m-d_{1}+d_{2}\right) \frac{T}{T+\tau}-d_{2} .
$$

Proof. By Theorem 2 in [11],

$$
\lambda=\lim _{n} \frac{1}{n} \log r\left(M\left(T_{n}, \tau_{n}\right) M\left(T_{n-1}, \tau_{n-1}\right) \ldots M\left(T_{1}, \tau_{1}\right)\right)
$$

It has been noted that the spectral radius of a matrix lies between the maximum and minimum column sums of a matrix. It is easily verified that the maximum (minimum) column sum of a product of matrices is not more (less) than the product of the maximum (minimum) column sums of each factor. Consequently, if $C_{i}\left(c_{i}\right)$ is the maximum (minimum) column sum of $M\left(T_{i}, \tau_{i}\right)$ then

$$
\prod_{i=1}^{n} c_{i} \leq r\left(M\left(T_{n}, \tau_{n}\right) M\left(T_{n-1}, \tau_{n-1}\right) \ldots M\left(T_{1}, \tau_{1}\right)\right) \leq \prod_{i=1}^{n} C_{i}
$$

As noted in Proposition 4,

$$
s e^{\left(m-d_{1}\right) T_{i}-d_{2} \tau_{i}} \leq c_{i} \leq C_{i} \leq e^{\left(m-d_{1}\right) T_{i}-d_{2} \tau_{i}}
$$

Consequently,

$$
\begin{aligned}
& \log s+\frac{1}{n} \sum_{i}\left(\left(m-d_{1}\right) T_{i}-d_{2} \tau_{i}\right) \leq \frac{1}{n} \log r\left(M\left(T_{n}, \tau_{n}\right) M\left(T_{n-1}, \tau_{n-1}\right) \ldots M\left(T_{1}, \tau_{1}\right)\right) \\
& \leq \frac{1}{n} \sum_{i}\left(\left(m-d_{1}\right) T_{i}-d_{2} \tau_{i}\right)
\end{aligned}
$$

On taking limits, we get

$$
\log s+\left(m-d_{1}\right) T-d_{2} \tau \leq \lambda \leq\left(m-d_{1}\right) T-d_{2} \tau .
$$

\section{Discussion}

We have constructed simple linear models of a dormancy-capable bacterial population consisting of cells that may transit between active and dormant 
states and which resides in a time-varying environment. Active cells grow while dormant cells suffer a lower removal rate. We have examined both periodic and randomly changing environments. The models differ in the switching strategies used to transit between dormant and active state. Our focus has been on determining the dependence of population fitness (dominant Lyapunov exponent) on these strategies as well as on environmental factors. More precisely, we focus on the dependence of the fitness on growth and death rates $m$ and $d_{1}>d_{2}$, on the duration of favorable and unfavorable periods $T_{1}$ and $T-T_{1}$, and on transit rates between dormant and active states $a, b$. Similar models have been studied by others $[16,17,3,4]$. Our work is distinguished by aiming for mathematically rigorous, analytical results, although we also use numerical calculations of fitness to explore parameter regions outside the reach of these results. Following Kussell [16] we have explored two idealized transition strategies between the active and dormant state. The responsive strategy presumes that bacteria can sense changes in the environment and switch to the most favorable state; it does so at rate $a$ for Dormant $\rightarrow$ Active during favorable periods and at rate $b$ for Active $\rightarrow$ Dormant during unfavorable periods. The stochastic strategy assumes organisms do not bother to monitor the environment but simply randomly transit between the two states at the rates just mentioned but independent of the environmental state. We compared the fitness of these two switching strategists relative to an organism incapable of dormancy (always active) which we referred to as "sleepless". Of course, it must be borne in mind that a responsive strategy is more expensive than a stochastic strategy since the former requires sensory apparatus.

Our analytical results are most complete in the case of a periodic environment with period $T$ in which the populations enjoy $T_{1}$ units of good times and $T-T_{1}$ units of bad times. We were able to obtain explicit bounds on the dominant Lyapunov exponent (Floquet multiplier) measuring fitness and comparisons between the fitnesses of the different switching strategists. Our results include the following:

(1) Both the responsive and stochastic switchers are more fit than sleepless when good times are rare $\left(T_{1} / T\right.$ is small).

(2) Responsive switcher is more fit than stochastic switcher when either good times are very rare or are very common.

(3) As expected, all fitnesses increase with increasing growth rate and de- 
crease with increasing death rates. Responsive fitness increases with cycle length $T$ with $T_{1}$ fixed and stochastic fitness increases with the duration of good times $T_{1}$ with $T$ fixed.

(4) There is a unique fast-switching limiting fitness for responsive switching but an entire interval of limiting potential fitnesses depending on the ratio $a / b$ for stochastic switching. The limiting responsive fitness exceeds any limiting value of stochastic switching as well as that of sleepless.

Numerical fitness calculations demonstrate that stochastic switcher can have higher fitness that either responsive switcher or sleepless when the duration of good times is in the moderate range. All possible relations among the fitnesses of the three phenotypes have been observed. Numerical calculations also show the surprising result that both stochastic and responsive fitness could initially decrease with increasing transit rates $a, b$ when their ratios are held fixed.

Our analytical results are meager in the case of a random environment. We assumed that good times and bad times alternated, that the duration of good times were selected independently from a fixed distribution and that the duration of bad times were selected independently from another fixed distribution. We showed that population fitness is well-defined by a dominant Lyapunov exponent using the theory of random dynamical systems [1] and the Random Perron-Frobenius Theorem $[2,11]$. Perhaps surprisingly, we obtained explicit lower and upper estimates of the fitness of both switching strategies which exactly agree with those obtained in the periodic case where one replaces the mean value of the good-times duration for $T_{1}$ and the sum of the two means for $T$. Indeed, the somewhat sharper estimate in the responsive case allows us to obtain the same unique fast-switching limit fitness that we obtained in the periodic environment. These facts suggest that studying the periodically cycling environment is not the waste of time and effort one might guess. Perhaps some of the other conclusions may extend from the periodic to the random environment.

\section{References}

[1] L. Arnold, "Random Dynamical Systems", Springer, 1998 
[2] L. Arnold, V.M.Gundlach, L.Demetrius, "Evolutionary Formalism for Products of Positive Random Matrices", The Annals of Applied Probability 4:3, 859-901. 1994

[3] N.Q. Balaban, J. Merrin,R. Chait, L. Kowalik, S.Leibler, Bacterial Peristence as a Phenotypic Switch, Science, 10 Sept. 2004, v. 305, 16221625.

[4] M. Bär, J. von Hardenberg, E. Meron and A. Provenzale, "Modelling the survival of bacteria in drylands: the advantage of being dormant", Proc. R. Soc. Lond. B(2002) 269, 937-942.

[5] A. Berman and R. Plemmons, Nonnegative matrices in the mathematical sciences, (Academic Press, New York 1979).

[6] A. Berman, M. Neumann, R. Stern, Nonnegative Matrices in Dynamical Systems, Wiley, New York 1989.

[7] R. Durrett, "Probability: Theory And Examples", Brooks/Cole, 1991.

[8] S. Elaydi, An Introduction to Difference Equations, 3rd ed., Springer, New York 2005.

[9] J. Grasman, Asymptotic Methods for Relaxation Oscillations and Applications, Springer-Verlag, Applied math. Sci. 63, New York, 1987.

[10] K.P. Hadeler and T. Hillen, Coupled Dynamics and Quiescent Phases, In: Math Everywhere- Deterministic and Stochastic Modelling in Biomedicine, Economy and Industry. Proc. Conf. Milano 2005, Springer 2006.

[11] H. Hennion, Limit Theorems for Products of Positive Random Matrices, Annals of Prob. 25, 1997, 1545-1587.

[12] A. Kaprelyants, J.C.Gottschal and D. Kell, "Dormancy in nonsporulating bacteria", FEMS Microbiology Reviews (1993), 271-286

[13] D.B. Kell, A.S Kaprelyants, D.H.Weichart, C.R. Harwood, M.R.Barer, Viability and activity in readily culturable bacteria: a review and discussion of the practical issues, A. van Leeuwenhoek 73, 1998, 169-187. 
[14] T.Malik, H.L. Smith "A Resource-Based Model of Microbial Quiescence", J. Math. Biol. Springer Verlag 2006, 53:2 231-252

[15] E.R. Lewis, "Network models in population biology", Springer-Verlag, 1977

[16] E.Kussell, R.Kishony, N.Q.Balaban and S.Leibler, "Bacterial Persistence: A Model of Survival in Changing Environments", Genetics, 169, April 2005, 1807-1814

[17] E.Kussell and S.Leibler, "Phenotypic Diversity, Population Growth, and Information in Fluctuating Environments, Science vol 309, Sept. 2005, 2075-78.

[18] H.L. Smith, and P. Waltman, The Theory of the Chemostat, Cambridge University Press, New York (1995).

[19] H.R. Thieme, Mathematics in Population Biology, Princeton University Press, Princeton 2003

[20] E. Seneta, Non-Negative Matrices, Wiley \& Sons, New York, 1973. 